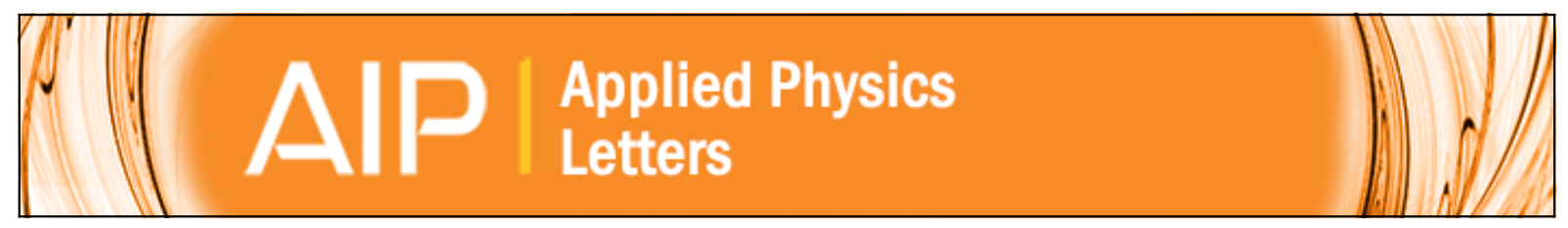

\title{
Heteroepitaxy of AIGaN on bulk AIN substrates for deep ultraviolet light emitting diodes
}

Z. Ren, Q. Sun, S.-Y. Kwon, J. Han, K. Davitt, Y. K. Song, A. V. Nurmikko, H.-K. Cho, W. Liu, J. A. Smart, and

L. J. Schowalter

Citation: Applied Physics Letters 91, 051116 (2007); doi: 10.1063/1.2766841

View online: http://dx.doi.org/10.1063/1.2766841

View Table of Contents: http://scitation.aip.org/content/aip/journal/apl/91/5?ver=pdfcov

Published by the AIP Publishing

\section{Articles you may be interested in}

Ductile relaxation in cracked metal-organic chemical-vapor-deposition-grown AIGaN films on GaN

J. Appl. Phys. 97, 123504 (2005); 10.1063/1.1929856

On the microstructure of $\mathrm{Al} \times \mathrm{Ga} 1 \times \mathrm{N}$ layers grown on $6 \mathrm{H}-\mathrm{SiC}(0001)$ substrates

J. Appl. Phys. 97, 083501 (2005); 10.1063/1.1861152

Evolution of stress in GaN heteroepitaxy on AIN Si ( 111 ) : From hydrostatic compressive to biaxial tensile Appl. Phys. Lett. 85, 3441 (2004); 10.1063/1.1808237

Control and elimination of cracking of $\mathrm{AlGaN}$ using low-temperature AIGaN interlayers

Appl. Phys. Lett. 78, 67 (2001); 10.1063/1.1336812

High optical quality AllnGaN by metalorganic chemical vapor deposition

Appl. Phys. Lett. 75, 3315 (1999); 10.1063/1.125336

\section{A|P|Applied Physics}

AIP Letters

is pleased to announce Reuben Collins as its new Editor-in-Chief

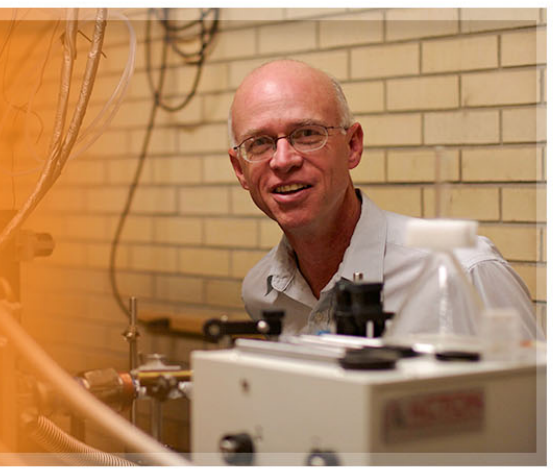




\title{
Heteroepitaxy of AIGaN on bulk AIN substrates for deep ultraviolet light emitting diodes
}

\author{
Z. Ren, Q. Sun, S.-Y. Kwon, and J. Han ${ }^{\text {a) }}$ \\ Department of Electrical Engineering, Yale University, New Haven, Connecticut 06520 \\ K. Davitt, Y. K. Song, and A. V. Nurmikko \\ Division of Engineering, Brown University, Providence, Rhode Island 02192 \\ H.-K. Cho \\ School of Advanced Materials Science \& Engineering, Sungkyunkwan University, Gyeonggi-do 440-746, \\ Korea
}

W. Liu, J. A. Smart, and L. J. Schowalter

Crystal IS, Green Island, New York 12183

(Received 10 May 2007; accepted 5 July 2007; published online 1 August 2007)

\begin{abstract}
The authors report the growth of AlGaN epilayers and deep ultraviolet (UV) light emitting diodes (LEDs) on bulk AlN substrates. Heteroepitaxial nucleation and strain relaxation are studied through controlled growth interruptions. Due to a low density of preexisting dislocations in bulk AlN, the compressive strain during $\mathrm{AlGaN}$ heteroepitaxy cannot be relieved effectively. The built-up of strain energy eventually induces either an elastic surface roughening or plastic deformation via generation and inclination of dislocations, depending on the stressor interlayers and growth parameters used. AlGaN LEDs on bulk AlN exhibit noticeable improvements in performance over those on sapphire, pointing to a promising substrate platform for III-nitride UV optoelectronics. (c) 2007 American Institute of Physics. [DOI: 10.1063/1.2766841]
\end{abstract}

Most of AlGaN deep ultraviolet $(\lambda<300 \mathrm{~nm})$ light emitting diodes ${ }^{1-4}$ (UV LEDs) are grown on mismatched sapphire substrates which limit the ultimate device performance due to a high density of dislocations (mid- $10^{9}-10^{10} \mathrm{~cm}^{-2}$ ). Low-defect bulk AlN substrates ${ }^{6}$ have attracted much attention because of its close matching to AlGaN in lattice parameters and thermal expansion coefficients, in addition to a much higher thermal conductivity. Preliminary demonstrations of III-nitride devices on AlN substrates ${ }^{7,8}$ have been reported even though little is known about the heteroepitaxial process. In this letter, we report a study of the growth of AlGaN on bulk AlN substrates toward the realization of high-performance UV LEDs. Structural and morphological studies during growth evolution indicate the compressive strain between $\mathrm{Al}_{0.5} \mathrm{Ga}_{0.5} \mathrm{~N}$ and $\mathrm{AlN}(\sim 1.2 \%)$ is not relaxed effectively due to the sparseness of preexisting dislocations $\left(<10^{5} \mathrm{~cm}^{-2}\right)$, resulting in alternative pathways of elastic surface roughening or misfit dislocating that resemble the $\mathrm{SiGe} / \mathrm{Si}$ and InGaAs/GaAs systems. ${ }^{9}$ The interplay between elastic and plastic relaxations leads to a trade-off in optimizing structural and morphological qualities. Using appropriate stressor interlayers and growth parameters for the optimum structural quality, deep UV AlGaN LEDs were grown on bulk AlN which exhibit distinct improvements in light output, device resistance, and thermal management under highlevel injection, suggesting that bulk AlN offers a promising route to high performance UV optoelectronics.

Growth was carried out by metal organic chemical vapor deposition (MOCVD) in a horizontal-flow reactor (Aixtron 200/4 HT-S) using trimethylgallium, trimethylaluminum, and ammonia $\left(\mathrm{NH}_{3}\right)$. Silane and meth- $\mathrm{Cp}_{2} \mathrm{Mg}$ were used as $n$ - and $p$-type dopants. Nominally $c$-plane AIN substrates

${ }^{a)}$ Electronic mail: jung.han@yale.edu $\left( \pm 0.5^{\circ}\right)$ were solvent degreased followed by wet etching. Prior to the homoepitaxial growth of AlN, the AlN substrates were annealed in situ under a mixture of $\mathrm{NH}_{3}$ and $\mathrm{H}_{2}$ at $1150{ }^{\circ} \mathrm{C} .{ }^{3}$ Deep UV AlGaN diodes with multiple-quantum wells active region ${ }^{3}$ were grown on top of $1 \mu \mathrm{m} n$-type $\mathrm{Al}_{0.50} \mathrm{Ga}_{0.50} \mathrm{~N}$ layer $\left([\mathrm{Si}] \sim 2 \times 10^{19} \mathrm{~cm}^{-3}\right)$. Design and growth of stress-management buffer layers between AlN and $\mathrm{Al}_{0.50} \mathrm{Ga}_{0.50} \mathrm{~N}$ layer will be discussed later. The Mg concentration on the $p$-side is maintained at an optimum level of around $3 \times 10^{19} \mathrm{~cm}^{-3} \cdot{ }^{10}$ Atomic force microscopy (AFM) was conducted using a Digital Instruments Nanoprobe III model with tapping mode. The Al composition and structural quality of $\mathrm{AlGaN}$ were determined by x-ray diffraction (XRD) using a triple-axis Bede D-1 diffractometer with symmetric and skewed symmetric scans. Transmission electron microscopy (TEM) experiments were performed in a JEOL 2010FX microscope operated at $200 \mathrm{kV}$ on samples. Cylindrical mesa-etched LED devices with optical apertures of 100 and $50 \mu \mathrm{m}$ diameters were fabricated and light was extracted with a procedure similar to our previous report. ${ }^{3}$ None of these devices has any additional heat sinking or light extraction schemes applied.

Homoepitaxy of AlN layers were examined first by AFM which indicates a smooth background with regularly spaced atomic steps (images not included). XRD rocking curve measurement of AlN typically exhibits full widths at half maximum (FWHMs) of $0.01^{\circ}$ and $0.02^{\circ}-0.03^{\circ}$ for $(0002)$ and (1012) diffractions, respectively. It was observed subsequently that direct growth of $\mathrm{Al}_{0.5} \mathrm{Ga}_{0.5} \mathrm{~N}(1 \mu \mathrm{m})$ on $\mathrm{AlN}$ homoepitaxial layers always results in a roughened morphology under AFM. To facilitate heteroepitaxial strain relaxation while preserving structural quality, we adopted a design of step-graded layers consisting of three superlattices (SLs 1, 2, and 3) with average $\mathrm{Al}$ compositions of $0.90,0.73$, and 0.57 , 

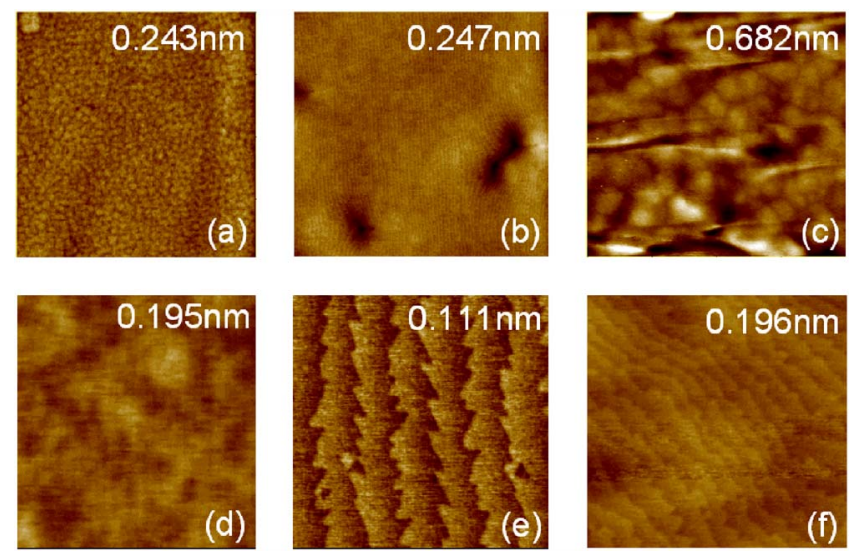

FIG. 1. (Color online) AFM images of high Al-content AlGaN superlattices on bulk AlN. The scan area of (a), (b), and (c) is $10 \times 10 \mu \mathrm{m}^{2}$ and (d), (e), and (f) $1 \times 1 \mu \mathrm{m}^{2}$. (a) and (d), (b) and (e), and (e) and (f) are taken after the growth of superlattices 1,2 , and 3, respectively. Root-mean-square (rms) roughness from each scan is labeled.

similar to the contemporary design in $\mathrm{SiGe} / \mathrm{Si}$ heteroepitaxy. ${ }^{11}$ Each SL is composed of ten periods of $\mathrm{Al}_{x} \mathrm{Ga}_{1-x} \mathrm{~N} \quad(150 \AA) / \mathrm{Al}_{y} \mathrm{Ga}_{1-y} \mathrm{~N} \quad(150 \AA) \quad(x / y=1.0 / 0.8$, $0.8 / 0.65$, and $0.65 / 0.50)$. Interrupted growth studies were carried out after each SL to reconstruct ex situ the heteronucleation process. Surface morphology after the growth of SL 1 [Figs. 1(a) and 1(d)] and SL 2 [Figs. 1(b) and 1(e)] indicates that pseudomorphic growth persists with an atomically smooth surface under a step-flow growth mode. The appearance of straight atomic steps of $1 \mathrm{ML}$ height $(\sim 2.5 \AA)$ with alternating saw-tooth features [Fig. 1(e)] reflects the difference in the step-edge atomic termination between two adjacent monolayers. ${ }^{12}$ There is one dangling

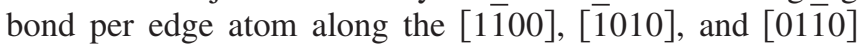
directions while there are two dangling bonds per edge atom along the $[10 \overline{1} 0],[0 \overline{1} 10]$, and $[\overline{1} 100]$ directions, leading to a faster step advance along the latter three directions. During the growth of SL 3, surface morphology undergoes a fundamental change with the appearance of large $(1-2 \mu \mathrm{m})$ plateaus or platelets separated by deep trenches or clifflike edges with a height of $\sim 100 \mathrm{~nm}$ [Fig. 1(c)] even though step flow is still maintained locally [Fig. 1(f)]. The deterioration of morphology at this stage is correlated with decay in in situ optical reflectance signal, suggesting that surface roughening on a scale of optical wavelength has occurred. ${ }^{13}$

Previous studies ${ }^{5,14,15}$ of AlGaN growth on sapphire or Si (111) substrates have shown that the compressive strain is effectively relaxed through the inclination of preexisting dislocations $\left(5 \times 10^{9}-3 \times 10^{10} \mathrm{~cm}^{-2}\right)$; good agreement was obtained based on an analytical model $\left(\varepsilon^{\mathrm{av}}=b h \tan (\theta) \rho / 4\right.$, with $\varepsilon^{\text {av }}$ the average relaxation, $b$ the Burgers vector, $h$ the film thickness, $\rho$ the dislocation density, and $\theta$ the dislocation inclination angle). ${ }^{14}$ However, due to a much lower density of preexisting dislocations $\left(<10^{5} \mathrm{~cm}^{-2}\right)$ in bulk AlN, the proposed model of dislocation inclination alone is insufficient in providing strain relaxation of $\mathrm{AlGaN}$ grown on bulk AlN. The observed surface roughening [Fig. 1(c)] beyond initial growth points to possibly an alternative pathway through elastic strain relaxation. Given the relatively low mobility of dislocations in III-nitride under typical growth conditions, ${ }^{16}$ the employment of step-graded buffer scheme contributes to a gradual built-up of compressive strain (SLs 1

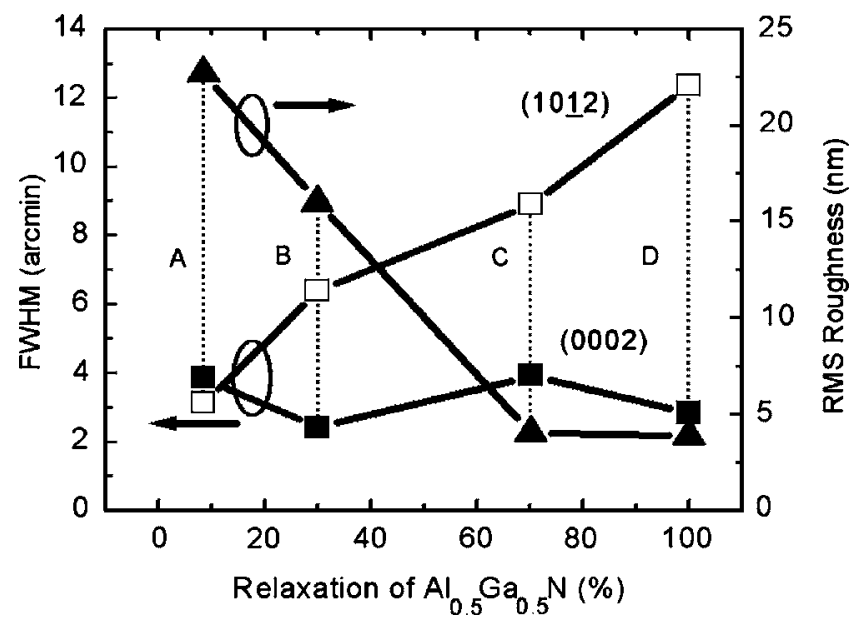

FIG. 2. Relations between surface RMS roughness (triangles) from AFM images $\left(20 \times 20 \mu \mathrm{m}^{2}\right)$, FWHMs of XRD rocking curves along (0002) (solid squares), (1012) (open squares) diffractions, and the degree of strain relaxation for $1 \mu \mathrm{m} \mathrm{Al}_{0.50} \mathrm{Ga}_{0.50} \mathrm{~N}$ grown on single superlattice buffers.

and 2) and consequently elastic relaxation (SL 3).

With a view of tailoring the nucleation mismatch and stress relaxation between AlN homoepitaxial layer and $\mathrm{Al}_{0.5} \mathrm{Ga}_{0.5} \mathrm{~N}$ layer, we tested a single superlattice design [AlN $\left.(150 \AA) / \mathrm{Al}_{0.50} \mathrm{Ga}_{0.50} \mathrm{~N} \quad(150 \AA) \times 10\right]$ as a stressor interlayer, ${ }^{17}$ during which the growth rate $(0.45-1.2 \mu \mathrm{m} / \mathrm{h})$ and V/III ratio (10-40) were varied to affect the heteroepitaxial evolution. Figure 2 shows the structural and morphological qualities of four $1-\mu \mathrm{m}$-thick $\mathrm{Al}_{0.5} \mathrm{Ga}_{0.5} \mathrm{~N}$ layers $(\mathrm{A}, \mathrm{B}$, $\mathrm{C}$, and D) as a function of the degree of relaxation determined by $x$-ray diffraction ${ }^{17}(8.6 \%, 30.0 \%, 75.2 \%$, and $100 \%$ for samples A, B, C, and D, respectively). An inverse relation between surface rms roughness and degree of relaxation indicates a complementary interplay between elastic and plastic relaxation mechanisms. For the samples with minimum relaxation (i.e., A), XRD rocking curve FWHMs of around 200 arc sec are observed along both (0002) and (10드) diffractions.

TEM analysis of the least and most relaxed samples (A and D) is shown in Figs. 3(a) and 3(b), respectively. The threading dislocation density in $\mathrm{Al}_{0.5} \mathrm{Ga}_{0.5} \mathrm{~N}$ layer of sample $\mathrm{A}$ is estimated to be one order of magnitude lower than that in sample D, which is in qualitative agreement with the XRD trend in Fig. $2 .{ }^{18} \mathrm{We}$ also note that as the degree of relaxation increases from $8.6 \%$ to $100 \%$, the observed maximum inclination angle of dislocations decreases accordingly from $\sim 60^{\circ}$ to $\sim 20^{\circ}$, pointing to the fact that grown-in strain is likely the driving force for dislocation inclination. Using the dislocation density deduced from XRD data as lower bounds

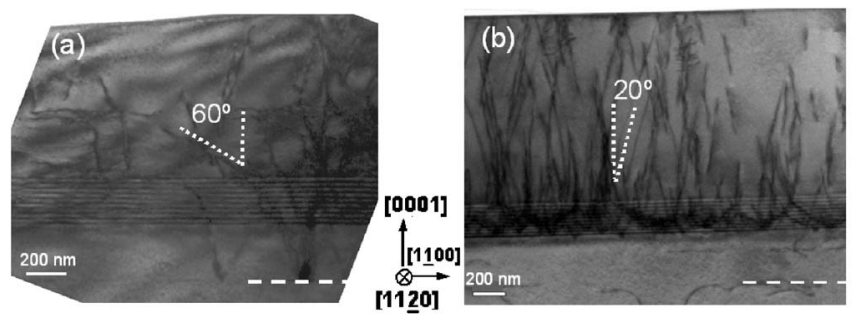

FIG. 3. Cross sectional TEM images of $\mathrm{Al}_{0.50} \mathrm{Ga}_{0.50} \mathrm{~N}$ layers on single $\mathrm{SL}$ buffer. (a) Least relaxed sample (sample A). (b) Most relaxed sample (sample $\mathrm{D}$ ). The dash lines indicate the beginning of AIN homoenitaxial layer. 

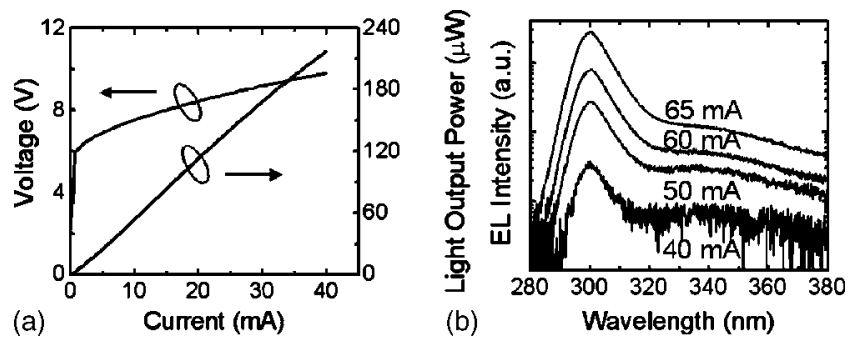

FIG. 4. Performance of deep UV LEDs on bulk AlN. (a) $L-I-V$ characteristics of $50 \mu \mathrm{m}$ diameter device. (b) EL spectra with different currents (from 40 to $65 \mathrm{~mA}$ ) for a $100 \mu \mathrm{m}$ diameter device.

and TEM as the upper, we estimated ${ }^{14}$ the strain relaxation through dislocation inclination to be in a range of 3-20\% and $15-50 \%$ for samples $\mathrm{A}$ and $\mathrm{D}$, respectively, compared to the values of $8.6 \%$ and $100 \%$ determined by XRD measurements (Fig. 2). The discrepancy in estimated and measured strain relaxation in sample D may be reconciled by noting the presence of a dense network of basal-plane misfit dislocations in the superlattice region, not observed in sample $\mathrm{A}$, with threading arms propagating into $\mathrm{Al}_{0.50} \mathrm{Ga}_{0.50} \mathrm{~N}$ layer [Fig. $3(\mathrm{~b})$. It is likely that the injection and glide of misfit dislocations during the superlattice growth provide an initial venue for strain relaxation; relaxation of the remaining strain proceeds through the inclination of threading arms. Generation and glide of misfit dislocations, a phenomenon not commonly observed in III-nitride growth, have been reported in InGaN heteroepitaxy on low-defect GaN layers. ${ }^{19}$ Understanding and control of the strain relaxation will be a crucial subject of investigation for III-nitride epitaxy on low-defect mismatched substrates.

Figure 4(a) shows the dc current-voltage and light output powers measured directly off the chip for $50 \mu \mathrm{m}$ diameter UV LED grown on AIN substrate with three step-graded SLs (SLs 1, 2, and 3). The electrical characteristics are markedly improved, compared to identical devices on sapphire, ${ }^{3}$ showing a sharp turn-on behavior and greater than $30 \%$ decrease in series resistance. Transmission line model method studies on the $n-\mathrm{Al}_{0.50} \mathrm{Ga}_{0.50} \mathrm{~N}$ contact layer indicate a sheet resistance of $160 \Omega / \square$, four to five times smaller than that typically achieved for LEDs grown on sapphire. A cw light output power of $210 \mu \mathrm{W}$ is achieved at $40 \mathrm{~mA}$, approximately four times higher than that from nominally identical devices on sapphire. ${ }^{3}$ A series of electroluminescence (EL) spectra at increasing current injection [Fig. 4(b)] illustrates a peak emission wavelength of $300 \mathrm{~nm}$ and a relatively small longwavelength contribution. Additionally, the light output power under high-level injection for device on AlN shows no thermal rollover, an improvement attributed to the much higher thermal conductivity of AlN $(\sim 3.2 \mathrm{~W} / \mathrm{cm} \mathrm{K})$ (Ref. 6) over sapphire. While the performance of our UV LEDs on AlN at present is still below the state-of-the-art devices on sapphire, ${ }^{1}$ the comparative study for LEDs on different substrates indeed highlight the importance of dislocation reduction and point to the potential of native (AIN) substrates in UV optoelectronics.

In summary, we have reported a study of AlGaN heteroepitaxial nucleation on low-dislocation AlN bulk substrates. Our study indicates that strain relaxation pathways can be altered with appropriate stressor interlayers and growth parameters; closely matched or slowly graded buffer layers preserve structural quality and postpone surface roughening while an intentionally mismatched buffer layer would facilitate rapid introduction and propagation of misfit dislocations after initial surface roughening. The driving force for surface roughing is mitigated after plastic strain relaxation, and surface smoothness improves subsequently. Performance of deep UV LEDs on bulk AIN exhibits distinct improvement over those on sapphire, pointing to a promising substrate platform for high performance III-nitride ultraviolet optoelectronics.

This work is supported by the DARPA SUVOS program under SPAWAR Systems Center Contract No. N66001-02-C80. S.-Y.K. acknowledges the support of the Korea Research Foundation Grant funded by Korea Government (MOEHRD, Basic Research Promotion Fund) (KRF-2005-214-D00315).

${ }^{1}$ X. Hu, J. Deng, J. P. Zhang, A. Lunev, Y. Bilenko, T. Katona, M. S. Shur, R. Gaska, M. Shatalov, and A. Khan, Phys. Status Solidi A 203, 1815 (2006).

${ }^{2}$ A. J. Fischer, A. A. Allerman, M. H. Crawford, K. H. A. Bogart, S. R. Lee, R. J. Kaplar, W. W. Chow, S. R. Kurtz, K. W. Fullmer, and J. J. Figiel, Appl. Phys. Lett. 84, 3394 (2004).

${ }^{3}$ Z. Ren, S.-R. Jeon, M. Gherasimova, G. Cui, J. Han, H. Peng, Y. K. Song, A. V. Nurmikko, L. Zhou, W. Goetz, M. Krames, and H.-K. Cho, Mater. Res. Soc. Symp. Proc. 831, E1.4 (2005).

${ }^{4}$ A. Hanlon, P. M. Pattison, J. F. Kaeding, R. Sharma, P. Fini, and S. Nakamura, Jpn. J. Appl. Phys., Part 2 42, L628 (2003).

${ }^{5}$ D. M. Follstaedt, S. R. Lee, P. P. Provencio, A. A. Allerman, J. A. Floro, and M. H. Crawford, Appl. Phys. Lett. 87, 121112 (2005).

${ }^{6}$ J. C. Rojo, G. A. Slack, K. Morgan, B. Raghothamachar, M. Dudley, and L. J. Schowalter, J. Cryst. Growth 231, 317 (2001).

${ }^{7}$ X. Hu, J. Deng, N. Pala, R. Gaska, M. S. Shur, C. Q. Chen, J. Yang, G. Simin, M. A. Khan, J. C. Rojo, and L. J. Schowalter, Appl. Phys. Lett. 82, 1299 (2003).

${ }^{8}$ T. Nishida, T. Makimoto, H. Saito, and T. Ban, Appl. Phys. Lett. 84, 1002 (2004).

${ }^{9}$ R. Hull and E. A. Stach, in Thin Films: Heteroepitaxial Systems, edited by W. K. Liu and M. B. Santos (World Scientific, New Jersey, 1999), Vol. 15, Chap. 7, pp. 299-367; B. A. Joyce and D. D. Vvedensky, ibid. (World Scientific, New Jersey, 1999) Vol. 15, Chap. 8, pp. 368-400.

${ }^{10}$ S.-R. Jeon, Z. Ren, G. Cui, J. Su, M. Gherasimova, J. Han, H.-K. Cho, and L. Zhou, Appl. Phys. Lett. 86, 082107 (2005).

${ }^{11}$ S. B. Samavedam and E. A. Fitzgerald, J. Appl. Phys. 81, 3108 (1997).

${ }^{12}$ M. H. Xie, S. M. Seutter, W. K. Zhu, L. X. Zheng, H. Wu, and S. Y. Tong, Phys. Rev. Lett. 82, 2749 (1999).

${ }^{13}$ T. B. Ng, J. Han, R. M. Biefeld, and M. V. Weckwerth, J. Electron. Mater. 27, 190 (1998).

${ }^{14}$ P. Cantu, F. Wu, P. Waltereit, S. Keller, A. E. Romanov, S. P. DenBaars, and J. S. Speck, J. Appl. Phys. 97, 103534 (2005).

${ }^{15}$ S. Raghavan, X. Weng, E. Dickey, and J. M. Redwing, Appl. Phys. Lett. 88, 041904 (2006).

${ }^{16}$ L. Sugiura, Appl. Phys. Lett. 70, 1317 (1997).

${ }^{17}$ J. Han, K. E. Waldrip, S. R. Lee, J. J. Figiel, S. J. Hearne, G. A. Petersen, and S. M. Myers, Appl. Phys. Lett. 78, 67 (2001).

${ }^{18}$ As was pointed out by S. R. Lee, A. M. West, A. A. Allerman, K. E. Waldrip, D. M. Follstaedt, P. P. Provencio, D. D. Koleske, and C. R. Abernathy, Appl. Phys. Lett. 86, 241904 (2005), the discrepancy of dislocation density measured by XRD and TEM depends on the specific nature of dislocations (randomly distributed or piled up in small angle grain boundaries). In our case, the assumption of randomly distributed dislocations is likely the reason for the underestimation of dislocation density using XRD by a factor of 5-10, compared to TEM results.

${ }^{19}$ S. Srinivasan, L. Geng, R. Liu, F. A. Ponce, Y. Narukawa, and S. Tanaka, Appl. Phys. Lett. 83, 5187 (2003). 